\title{
Pneumonia comunitária e pneumo- nia hospitalar em adultos
}

\section{Community-acquired pneumonia and nosocomial pneumonia in adults}

\author{
Pedro V. Schwartzmann ${ }^{1,3}$, Gustavo J. Volpe ${ }^{2,3}$, Fernando C. Vilar ${ }^{3,4}$, Julio C. Moriguti ${ }^{5}$
}

\begin{abstract}
RESUMO
Pneumonia bacteriana comunitária e hospitalar em adultos são problemas de saúde pública crescentes, com inúmeras internações anualmente e causas frequentes de morbimortalidade. A rápida caracterização do quadro clínico deve ser acompanhada de decisão quanto à necessidade de internação do paciente e o tratamento deve ser baseado na gravidade da apresentação e aspectos epidemiológicos. O acompanhamento clínico é imprescindível, tanto ambulatorialmente quanto na internação, atento aos critérios de falha terapêutica e necessidade de revisão da terapêutica inicialmente instituída. Particularmente na pneumonia hospitalar (nosocomial), o diagnóstico é um desafio e a análise do tempo do início do quadro é fundamental para o direcionamento empírico do tratamento. Ênfase na prevenção é uma tentativa de redução na frequência dos casos.
\end{abstract}

Palavras-chave: Pneumonia Bacteriana/diagnóstico. Pneumonia Bacteriana/transmissão. Infecção Hospitalar.

\section{Pneumonia comunitária}

\section{Definição}

Pneumonia pode ser definida como sinais e sintomas consistentes com infecção do trato respiratório baixo associado a novo infiltrado na radiografia de tórax, na ausência de outra explicação para tal. ${ }^{1}$ Pneumonia adquirida na comunidade é aquela que acomete o paciente fora do ambiente hospitalar ou que surge nas primeiras $48 \mathrm{~h}$ da internação hospitalar.

\section{Epidemiologia}

Há aproximadamente 4 milhões de novos casos de pneumonia adquirida na comunidade (PAC) nos Estados Unidos todos os anos, resultando em 1 milhão de hospitalizações, com grande impacto em custos e morbimortalidade. ${ }^{2}$ No Brasil, dados do DATASUS evidenciam a pneumonia como a maior causa de mortalidade dentre as doenças respiratórias e ocupa o $4^{\circ}$ lugar de mortalidade geral, excluindo-se as causas
1 Ex-Médico Assistente da Clínica Médica do Hospital Estadual de Ribeirão Preto.

2 Médico Assistente da Área de Clínica Médica do Hospital Estadual de Ribeirão Preto.

3 Pós-graduando da Area de Clínica Médica da FMRP-USP.

4 Médico Assistente do Serviço de Controle de Infecção Hospitalar do Hospital Estadual de Ribeirão Preto.

5 Diretor de Atividades Clínicas do Hospital Estadual de Ribeirão Preto e Professor Livre-Docente da Divisão de Clínica Médica Geral e Geriatria da FMRP-USP.
Correspondência: Julio Cesar Moriguti Hospital Estadual de Ribeirão Preto Avenida Independência, 4750. 14026-160- Ribeirão Preto - SP Telefone: $16-3602-7100$. 
externas. Estima-se a incidência mundial em 12 casos por 1000 habitantes/ano. ${ }^{3}$

\section{Etiologia}

Na maior parte dos casos, o germe responsável pelo quadro não é identificado. Sabe-se que o pneumococo é o germe mais frequentemente implicado na PAC, em todas as faixas etárias, sendo responsável por cerca de dois terços dos casos de pneumonias bacterianas da comunidade. ${ }^{4}$ Os demais agentes podem estar relacionados com as características epidemiológicas abaixo ${ }^{5}$ :

- Tabagismo/DPOC: H. influenzae e M. catarrhalis

- Casas de repouso: bacilos gram negativos, anaeróbios

- Dentes em mau estado de conservação: anaeróbios e bacilos gram negativos

- Bronquiectasias e fibrose cística: anaeróbios

- Obstrução brônquica: anaeróbios

- Uso drogas injetáveis: S. aureus, anaeróbios e pneumococo

- Alcoolismo: anaeróbios, pneumococo resistente à penicilina, bacilos gram negativos. Lembrar também de tuberculose

- Uso recente de antibiótico de largo espectro: Pseudomonas, bacilos gram negativos e pneumococo resistente à penicilina.

Podemos também avaliar a prevalência dos germes de acordo com a gravidade da pneumonia, com três divisões: ambulatorial, com necessidade de internação, mas não em UTI e com necessidade de internação em UTI (Tabela 1).

\section{Quadro clínico e diagnóstico}

Pacientes com pneumonia geralmente se apresentam com tosse (90\%), dispnéia (66\%), dor pleurítica (50\%), além de sintomas constitucionais, como queda do estado geral, febre, hiporexia, adinamia. Pneumonia pode ser diagnosticada por meio dos critérios abai$\mathrm{xo}^{2,3}$ :

- Sintomas de doença aguda do trato respiratório inferior: tosse e um ou mais dos seguintes - expectoração, dispnéia e/ou dor torácica;

- Achados focais ao exame físico: redução localizada do murmúrio vesicular, aumento do frêmito toracovocal, macicez à percussão, pectorilóquia, dentre outros;

- Pelo menos um achado sistêmico: confusão, cefaléia, sudorese, calafrios, mialgias, febre;

- Infiltrado radiológico não presente previamente;

- Exclusão de outras causas que resultem em achados semelhantes.

Em pacientes idosos, esses sinais/sintomas podem não estar presentes, a apresentação clínica nos idosos manifesta-se pode manifestar por alteração abrupta da capacidade funcional, confusão mental e descompensação de doença previamente estável (como DPOC e ICC $)^{3}$, necessitando de alto nível de suspeição para diagnóstico mais precoce e correto tratamento.

\section{Radiografia de tórax}

A radiografia de tórax é indispensável tanto para o diagnóstico como para avaliação de gravidade, bem como para identificar condições coexistentes como derrame pleural, cavitações, número de lobos acometidos e obstrução brônquica (complicações); acompanhar evolução e resposta ao tratamento ${ }^{3}$, principalmente naqueles pacientes não respondedores à terapêutica inicial.

\section{Hemograma}

O exame hematológico complementa a avaliação clínica, e pode ser útil na caracterização de gravi-

\section{Tabela 1}

Prevalência de germes em ordem decrescente de frequência de acordo com a gravidade: ambulatorial, internação em enfermaria e internação em UTI

\begin{tabular}{lll}
\hline Ambulatorial & Enfermaria & UTI(Grave) \\
\hline S. pneumoniae & S. pneumoniae & S. pneumoniae \\
M. pneumoniae & M. pneumoniae & S. aureus \\
C. pneumoniae & C. pneumoniae & H. influenzae \\
Vírus respiratórios & H. influenzae & Legionella spp \\
H. influenzae & Legionella spp & Bacilos gram negativos \\
& Aspiração & \\
& Vírus Respiratórios & \\
\hline
\end{tabular}


dade quando há critérios para SIRS (Síndrome da Resposta Inflamatória Sistêmica) no leucograma: leucocitose $(>12.000)$ ou leucopenia $(<4000)$ ou mais de $10 \%$ de formas jovens.

\section{Gasometria arterial}

$\mathrm{Na}$ ausência de doença pulmonar crônica, a saturação de oxigênio abaixo de $90 \%$, indica maior gravidade (3), necessitando de suplementação de oxigênio. Na persistência de valores baixos apesar de $4 \mathrm{~L} / \mathrm{min}$ de oxigênio (suplementação de oxigênio por de cateter nasal) considerar internação em terapia intensiva.

\section{Hemocultura}

O atual consenso brasileiro de pneumonia recomenda a solicitação de hemoculturas em pacientes com pneumonia considerada grave (CURB- $65 \geq 3$ e PORT $\geq$ IV - vide descrição abaixo) e pacientes não respondedores ao tratamento clínico inicial. A título de memorização, a solicitação de hemocultura deve ser realizada quando resultar em alteração significativa do esquema antimicrobiano empírico, levando-se em conta aspectos clínicos e epidemiológicos. ${ }^{5}$ Algumas indicações de investigação adicional são: indicação de UTI, falência de tratamento ambulatorial, cavitações, leucopenia, alcoolismo, doença hepática crônica grave, doença pulmonar grave, asplenia e derrame pleural. ${ }^{5}$

\section{Escarro}

A análise e cultura do escarro são controversas ${ }^{6}$, em virtude da dificuldade na obtenção de amostras adequadas e na qualidade da análise e dos testes realizados pelo laboratório. Um estudo prospectivo avaliou o uso do escarro como ferramenta de esclarecimento diagnóstico e evidenciou que amostras adequadas de escarro foram obtidas somente em cerca de $16 \%$ dos casos. ${ }^{6}$ Uma metanálise avaliou 12 estudos quanto à sensibilidade e especificidade da análise do escarro: a sensibilidade comparada à cultura do escarro variou de $15-100 \%$ e a especificidade de 11 $100 \%{ }^{7}$

O guideline de 2007 da IDSA/ATS ${ }^{5}$ recomenda que a análise pré-tratamento do escarro deve ser realizada somente se for possível a obtenção de amostra de boa qualidade (adequada quantidade, com presença de polimorfonucleares e baixo número de células epiteliais escamosas; processamento para cultura deve ser realizado em até 2 horas, transporte rápido e análise de acordo com normas internacionais) nos seguin- tes casos: necessidade de UTI; falência de tratamento ambulatorial; lesões cavitárias; uso abusivo de álcool; doença obstrutiva grave ou doença estrutural pulmonar e derrame pleural.

\section{Avaliação da gravidade e critérios para internação}

A rápida caracterização do quadro clínico deve ser acompanhada de decisão quanto à necessidade de internação do paciente - avaliação da gravidade do quadro, doenças associadas, condição social e aderência são questões importantes e devem ser ponderadas para o manejo clínico. ${ }^{4}$

Nesse contexto, também o reconhecimento de fatores de risco para maior mortalidade como idade avançada, comorbidades (insuficiência cardíaca, neoplasia, doença coronariana, alcoolismo) e anormalidades no exame físico e radiológico, devem nortear o julgamento clínico.

Vários critérios foram propostos para analisar a necessidade de internação, como o PORT (Patient Outcome Research Team) - também conhecido como escore de gravidade de pneumonia - e, mais recentemente, o CURB-65 (proposto pela British Thoracic Society). No escore de gravidade de pneumonia (PORT), pontos são atribuídos aos muitos critérios analisados (Tabela 2) e, de acordo com o número de pontos obtidos, o paciente é alocado em uma das cinco possíveis estratificações de risco (Tabela 3), o que servirá como guia para manejo ambulatorial ou internado e quantificará a gravidade (mortalidade). ${ }^{3}$ Como são muitos aspectos a serem analisados, apesar de validada em muitos estudos, sua aplicabilidade é mais complexa. ${ }^{2}$ Para facilitar a interpretação do PORT, foi criado um fluxograma simplificado (Figura 1).

O critério acima descrito (PORT, também chamado de Pneumonia Severity Index), adotado pela American Thoracic Society (EUA), tem ênfase na identificação de pacientes de baixo risco, nos quais o tratamento ambulatorial seria a escolha. ${ }^{1}$

Já o critério CURB-65, designado pela British Thoracic Society, tem ênfase na identificação de pacientes com pneumonia comunitária grave, nos quais o tratamento hospitalar é mandatório. Pela facilidade de aplicação e memorização, o CURB-65 tem sido cada vez mais utilizado. Consiste na atribuição de pontos para os seguintes critérios: C - nível de consciência; $\mathrm{U}$ - uréia $>50 \mathrm{mg} / \mathrm{dl} ; \mathrm{R}$ - frequência respiratória $\geq$ 30 ipm; B (Blood pressure), PAS $<90 \mathrm{mmHg}$ e/ou 


\section{Tabela 2}

Escore de gravidade de pneumonia (PORT)

$\begin{array}{lc}\text { Idade } & 1 \text { ponto para cada ano } \\ \text { Sexo feminino } & -10 \\ \text { Reside em abrigo } & +10 \\ \text { Doença neoplásica } & +30 \\ \text { Doença hepática } & +20 \\ \text { Insuficiência cardíaca } & +10 \\ \text { Doença cerebrovascular } & +10 \\ \text { Doença renal } & +10 \\ \text { Confusão mental } & +20 \\ \text { FR } \geq 30 \text { ipm } & +20 \\ \text { PAS }<90 \mathrm{mmHg} & +20 \\ \text { Temperatura } \geq 40 \text { ou }<35^{\circ} \mathrm{C} & +15 \\ \mathrm{FC} \geq 125 \mathrm{bpm} & +10 \\ \mathrm{pH}<7,35 & +30 \\ \text { Uréia } \geq 30 \mathrm{mg} \% & +20 \\ \mathrm{Sódio}<130 \mathrm{mEq} / \mathrm{L} & +20 \\ \mathrm{Glicose}>250 \mathrm{mg} \% & +10 \\ \mathrm{Hematócrito}<30 \% & +10 \\ \mathrm{PaO}{ }_{2}<60 \mathrm{mmHg} \text { ou } \mathrm{SatO}_{2}<90 \% & +10 \\ \text { Derrame pleural } & +10\end{array}$

\section{PACIENTE TEM MAIS DE 50 ANOS DE IDADE?}

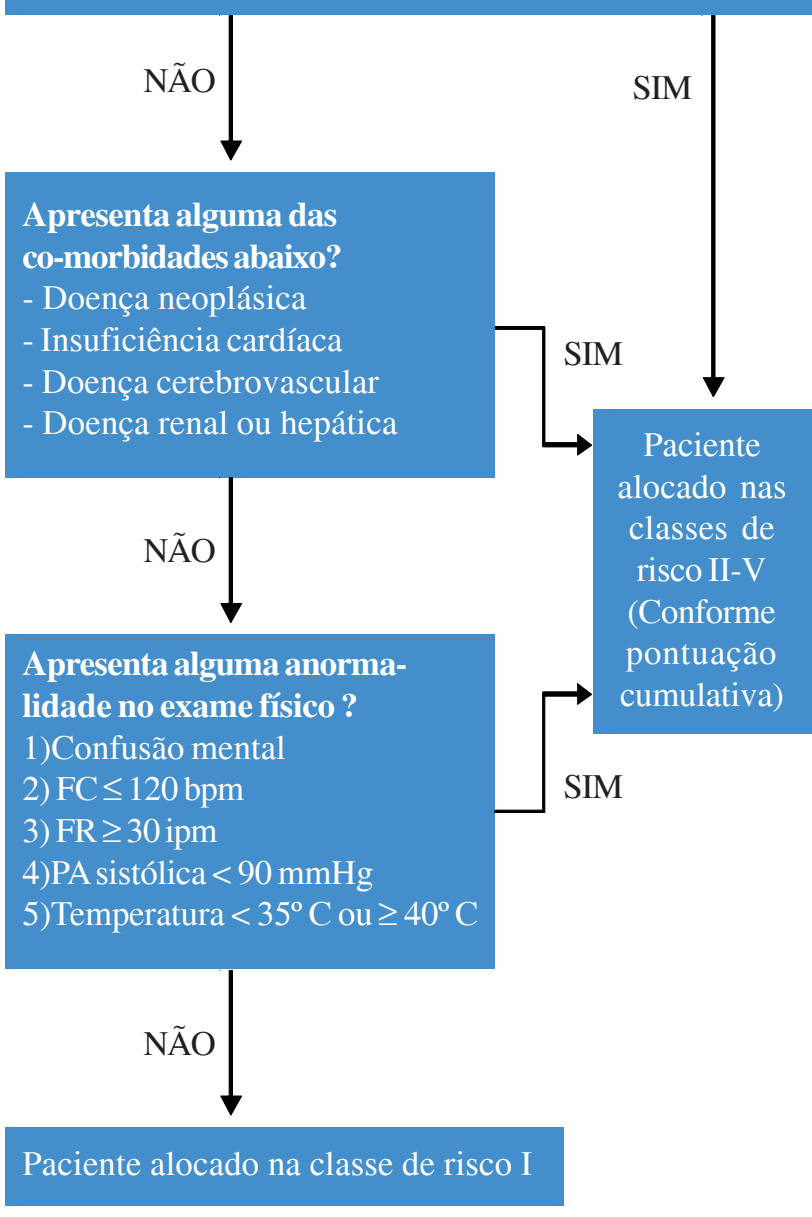

Figura 1. Abordagem simplificada ao Escore de PORT.

Tabela 3

Interpretação do Escore de PORT - soma dos pontos e decisão quanto à internação.

\begin{tabular}{lclcl}
\hline Risco & Classe & Pontos & Mortalidade (\%) & Tratamento \\
\hline Baixo & I & Algoritmo & 0,1 & Ambulatorial \\
Baixo & II & $\leq 70$ & 0,6 & Ambulatorial \\
Baixo & III & $71-90$ & 2,8 & Breve internação \\
Moderado & IV & $91-130$ & 8,2 & Hospitalar \\
Alto & V & $\geq 130$ & 29,2 & Hospitalar \\
\hline
\end{tabular}

PAD $\leq 60 \mathrm{mmHg}$; além de idade maior que 65 anos (Figura 2). Se a soma dos pontos estiver entre 0-1 há baixo risco de mortalidade, sendo preferível o tratamento ambulatorial; se totalizar 2 pontos, considerar tratamento hospitalar ; e, se $\geq 3$ pontos, o paciente é classificado como portador de pneumonia comunitária grave, com necessidade obrigatória de internação.
Os critérios apresentados devem fazer parte de um julgamento clínico amplo, que deve ponderar idade, comorbidades, achados de exame físico e radiografia de tórax, bem como fatores psicossociais, econômicos (Figura 2). Dentre os achados de exame físico, vale ressaltar a importância na identificação de SIRS, composto de dois ou mais critérios: 1) Taqui- 
cardia (FC > 90 bpm), 2) Taquipnéia (FR >20 ipm ou $\mathrm{pCO}_{2}<32 \mathrm{mmHg}$ ), 3) Febre ou hipotermia; 4) Leucocitose, leucopenia ou mais de $10 \%$ de formas jovens no hemograma. Além disso, é considerado contraindicação absoluta ao tratamento ambulatorial qualquer uma das seguintes condições: hipoxemia (Sat<90\% em ar ambiente), instabilidade hemodinâmica, condição ativa com necessidade de hospitalização e incapacidade de tolerar antibioticoterapia via oral. Caso CURB $65 \leq 2$ (não seria internado se esse critério fosse usado isoladamente), analisar outros fatores na sequência, como COX (Comorbidades, Saturação de $\mathrm{O}_{2}$ e radiografia (Raio-X) com velamento bilateral), além da condição social, antes de optar por tratamento ambulatorial. Observe que o julgamento clínico não deve considerar isoladamente o critério CURB-65 na tomada da decisão quanto à necessidade de internação.

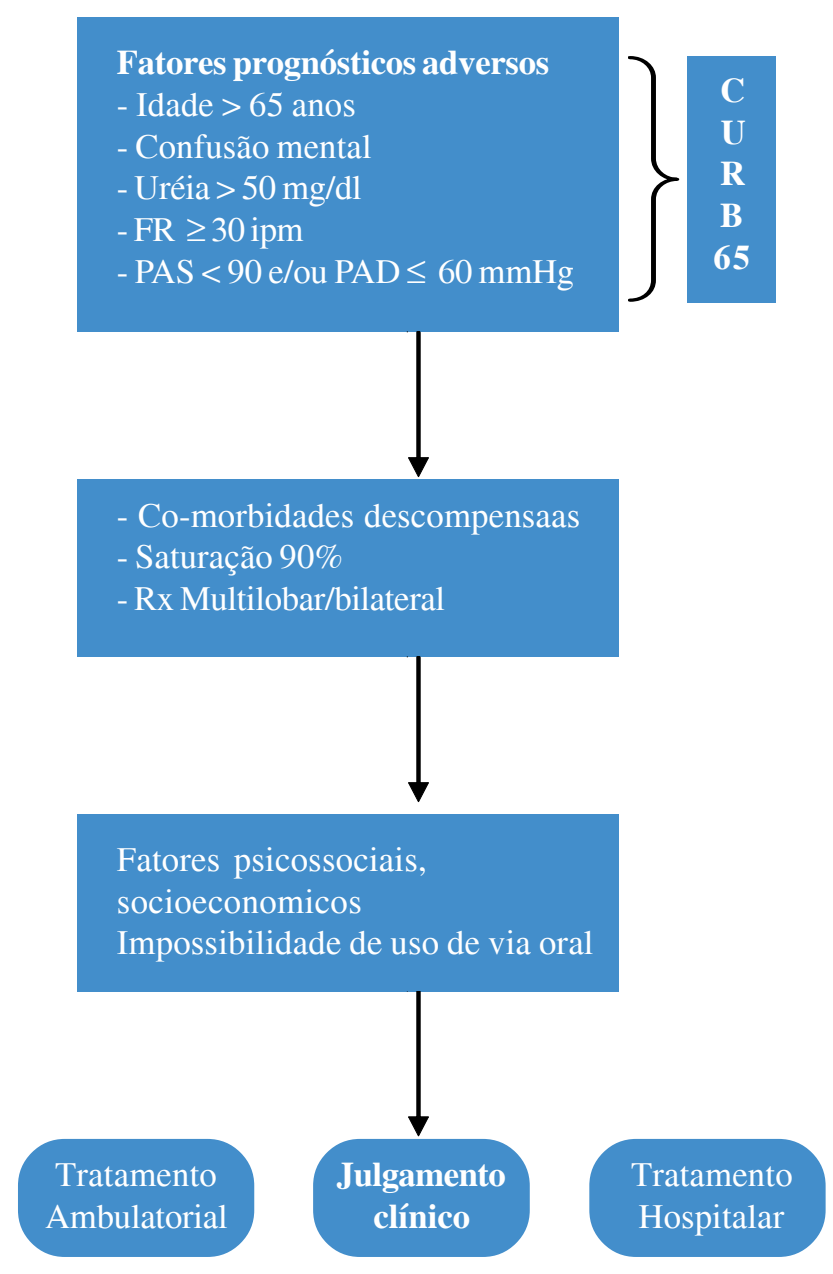

Figura 2. Fatores que influenciam o julgamento clínico quanto à necessidade do tratamento ambulatorial $\mathrm{x}$ internado.

\section{Indicação de Terapia Intensiva}

Com relação à pneumonia comunitária grave, a indicação de terapia intensiva é de fundamental importância, tendo em vista que a precocidade do reconhecimento pode melhorar o prognóstico e evolução. A antibioticoterapia deve ser iniciada em até 4 horas da chegada ao serviço hospitalar e, se os critérios abaixo forem preenchidos (1 critério maior ou 3 menores), o paciente deverá receber tratamento intensivo ${ }^{5}$ :

- Critérios maiores: 1) Instabilidade hemodinâmica com necessidade de vasopressores (choque séptico); 2) necessidade de ventilação mecânica ou ventilação não invasiva ou $\mathrm{FiO}_{2}>40 \%$ para manutenção de saturação de $\mathrm{O}_{2}>90 \%$.

- Critérios menores: 1) FR $\geq 30 \mathrm{ipm} ; 2) \mathrm{PaO}_{2} / \mathrm{FiO}_{2}$ $\leq 250$; 3) Infiltrado multilobar; 4) Confusão/Desorientação; 5) Uremia; 6) Leucopenia (GB < 4000 cels/ $\left.\mathrm{mm}^{3}\right)$; 7) Trombocitopenia $\left(<100.000\right.$ cels $\left./ \mathrm{mm}^{3}\right)$; 8) Hipotermia; 9) Hipotensão necessitando de reposição volêmica agressiva.

\section{Tratamento}

A terapêutica antimicrobiana é tipicamente iniciada de forma empírica, uma vez que a identificação do germe responsável não ocorre em grande parte dos casos. A seleção da terapêutica é baseada em alguns fatores: o patógeno mais comum em cada situação, fatores que aumentam o risco para determinados patógenos (Tabela 4), co-morbidades, estratificação de risco do caso (CURB 65 e escore de PORT) e efeitos adversos dos antibióticos (como alergia e causa de estado confusional em idosos, marcadamente com o uso de quinolonas nesse grupo de pacientes). A indicação de internação é baseada em julgamento clínico, conforme descrito acima.

A terapêutica empírica inicial deve ser modificada quando houver isolamento do patógeno em cultura, levando à redução do espectro antimicrobiano de acordo com o perfil de sensibilidade.

Qualquer derrame pleural significativo (acima de $1 \mathrm{~cm}$ na radiografia de decúbito lateral) deve ser puncionado para descartar empiema e derrame parapneumônico complicado, que demandam drenagem torácica.

\section{Pacientes ambulatoriais}

Estudos orientando o tratamento em pacientes ambulatoriais não são suficientes para recomendação 


\section{Tabela 4}

Patógenos mais comuns em cada situação e fatores que aumentam o risco de infecção por patógenos específicos

\begin{tabular}{ll} 
Pneumoco resistente & - Idade $>65$ anos \\
& - Terapia prévia com beta-lactâmico nos últimos 3 meses \\
& - Pacientes imunossuprimidos (doenças e/ou uso de corticosteróides) \\
& - Múltiplas co-morbidades \\
Bacilos gram negativos & - Pacientes de casas de repouso \\
& - Doença cardiopulmonar \\
& - Múltiplas comorbidades \\
& - Uso recente de antibióticos \\
& - Doença pulmonar estrutural \\
Pseudomonas aeruginosa & - Uso corticosteróides (>10mg/dia) \\
& - Uso de antibióticos de largo espectro por mais de 7 dias no último mês \\
& - Desnutrição \\
\hline
\end{tabular}

baseada em evidências. ${ }^{3}$ Os pacientes com PAC sem necessidade de internação são tratados de forma empírica, buscando um regime antimicrobiano contra os patógenos mais comuns nessa população (S. pneumoniae, M. pneumoniae, C. pneumoniae e $H$. influenzae). De acordo com as diretrizes brasileiras e americanas, um macrolídeo é recomendado como monoterapia para tratamento de pacientes ambulatoriais previamente sadios (azitromicina, claritromicina).

Macrolídeo associado a beta-lactâmico ou fluoroquinolona sozinha são recomendados para tratamento inicial empírico de pacientes com comorbidades que não preenchem critérios para internação (DPOC, ICC, diabetes, nefropatia e neoplasias). Betalactâmico com inibidor de beta-lactamase deve ser de escolha quando houver suspeita de aspiração e infecção por anaeróbios. Em caso de alergia à penicilina, utilizar clindamicina.

A duração do tratamento deve ser baseada na resposta ao tratamento, doenças associadas e complicações. ${ }^{4}$ Em geral, recomenda-se o tratamento até que o paciente se torne afebril por 72 horas (pneumonias por pneumoco); 7-14 dias no caso de germes atípicos. Se o paciente estiver afebril após 48-72 horas do início do tratamento, o período poderá ser encurtado para 5 dias; do contrário, o tratamento deverá se estender até 7-10 dias.

\section{Pacientes internados (enfermaria)}

A terapêutica empírica em pacientes com necessidade de internação será orientada de acordo com o critério de gravidade na admissão, comorbidades e busca de fatores que aumentam o risco de infecção por patógenos específicos (Tabela 4).

A primeira dose do antibiótico deve ser realizada em até 4 horas da admissão hospitalar, sempre por via parenteral. A escolha da terapêutica inicial tem ampla variação quando se analisa as recomendações das entidades americanas, européias e canadenses. A recomendação do European Respiratory Society e do consenso brasileiro é o uso isolado de quinolona respiratória (levofloxacino, moxifloxacino ou gatifloxacino) ou uso isolado ou combinado com macrolídeo das seguintes drogas: beta-lactâmico associado à betalactamase ou cefalosporinas de segunda/terceira geração (cefuroxima, cefotaxima, ceftriaxone). A associação com macrolídeo deve levar em conta comorbidades e gravidade na admissão (Tabela 5).

\section{Pacientes internados na UTI (pneumonia co- munitária grave)}

$\mathrm{O}$ tratamento de pacientes sem fatores de risco com PAC grave, sem risco para infecção por Pseudomonas, deve ser realizado com base no tratamento de pacientes internados na enfermaria (beta-lactâmico associado à beta-lactamase ou cefalosporinas de segunda/terceira geração combinado com macrolídeo ou quinolona respiratória isolada). Para pacientes com fatores de risco para infecção por Pseudomonas (Tabela 5), a recomendação do consenso brasileiro é o uso de agente antipseudomonas + ciprofloxacino ou agente antipseudomonas + aminoglicosídeo + fluoroquinolona ou macrolídeo. 


\section{Tabela 5}

Tratamento inicial empírico e parenteral de pacientes internados em enfermaria.

\begin{tabular}{|c|c|}
\hline Local de tratamento & Recomendação Terapêutica \\
\hline $\begin{array}{l}\text { AMBULATORIAL } \\
\text { Previamente sadio }\end{array}$ & $\begin{array}{l}\text { Macrolídeo (azitromicina } 500 \mathrm{mg} \text { VO } 1 \text { x/dia por } 5 \text { dias; } \\
\text { claritromicina } 500 \mathrm{mg} \text { VO 12/12 horas } 7-10 \text { dias); } \\
\text { se idade }<60 \text { anos, avaliar amoxicilina } 500 \mathrm{mg} \text { VO } 8 / 8 \text { horas }\end{array}$ \\
\hline $\begin{array}{l}\text { Terapia antimicrobiana recente } \\
\text { ou doenças associadas }\end{array}$ & $\begin{array}{l}\text { Levofloxacino } 500 \mathrm{mg} \text { VO } 1 \mathrm{x} / \text { dia por } 7 \text { dias } \\
\text { OU gatifloxacino } 400 \mathrm{mg} \text { VO } 1 \mathrm{x} / \text { dia por } 7 \text { dias } \\
\text { OU moxifloxacino } 400 \mathrm{mg} \text { VO } 1 \mathrm{x} / \text { dia por } 7 \text { dias } \\
\text { OU amoxicilina-clavulanato } 500 \mathrm{mg} \text { VO } 8 / 8 \text { horas + macrolídeo }\end{array}$ \\
\hline
\end{tabular}

Suspeita de aspiração

OU cefuroxima $500 \mathrm{mg} \mathrm{VO} 2 \mathrm{x} / \mathrm{dia}+$ macrolídeo

Amoxicilina-clavulanato $500 \mathrm{mg} \mathrm{VO} 8 / 8 \mathrm{hs}$

OU Clindamicina $600 \mathrm{mg}$ VO 6/6 horas

ENFERMARIA

Levofloxacino $500 \mathrm{mg}$ EV 1x/dia

OU amoxicilina-clavulanato $1 \mathrm{G} \mathrm{EV} \mathrm{8/8} \mathrm{horas} \mathrm{+} \mathrm{macrolídeo}$

TERAPIAINTENSIVA

Sem risco para $P$. aeruginosa

Com risco para P. Aeruginosa
Beta-lactâmico + macrolídeo ou quinolona

Agente antipseudomonas + ciprofloxacino

OU agente antipseudomonas + aminoglicosídeo + quinolona ou macrolídeo

\section{Critérios para transicionar antibioticoterapia para via oral}

A conversão da antibioticoterapia de endovenoso para via oral deve ser realizada quando o paciente preencher os seguintes critérios: deglutição adequada, sinais vitais estáveis por mais de 24 horas (ausência de febre, FC $<100$ bpm, FR <24 ipm, Pressão arterial sistólica $>90 \mathrm{mmHg}$ ) e adequado nível de consciência.

Quando paciente estiver estável e com boa aceitação de antibioticoterapia via oral, os critérios para alta são: adequado nível de consciência, possibilidade de uso de medicação em domícilio (condição social), possibilidade de manter adequado nível de nutrição e hidratação e se não houver nenhuma outra condição clínica ativa ou problema psicossocial que necessite prolongar a internação.

\section{Pacientes não respondedores à terapêutica empírica inicial}

Todos os pacientes devem ser reavaliados quanto à falha terapêutica, mesmo os pacientes alocados para tratamento ambulatorial. A imagem radiológica não deve ser utilizada como critério isolado, dado persistência das alterações radiológicas por até 7 sema- nas mesmo em pacientes com boa resposta clínica. ${ }^{5}$ Os principais fatores relacionados à falha terapêutica estão na Figura 3.

Falha terapêutica pode ser precoce (ausência de resposta ou piora do quadro clínico e radiológico em 48-72 horas) ou tardia (piora do quadro após 72 horas). Se não há explicação evidente para resposta demorada ou deterioração clínica, quatro principais fatores devem ser avaliados:

1) Seleção antimicrobiana inadequada (checar se microorganismo resistente à droga ou não coberto no esquema inicial; considerar pneumococo resistente, presença de fatores de risco para infecção por Pseudomonas aeruginosa ou S.aureus);

2) Patógenos não usuais: o diagnóstico diferencial inclui tuberculore, micobacteriose atípica, fungos endêmicos, pneumocistose. Muitas vezes o paciente apresenta pneumonia que aparentemente melhora (sobretudo se em uso de quinolona ou claritromicina) e evoluiu com piora do quadro - considerar tuberculose, nocardiose e verificar fatores de risco para imunossupressão como infecção pelo HIV, agentes imunossupressores e colagenoses.

3) Complicações: complicações extrapulmonares ocorrem em cerca de $10 \%$ dos pacientes com pneu- 


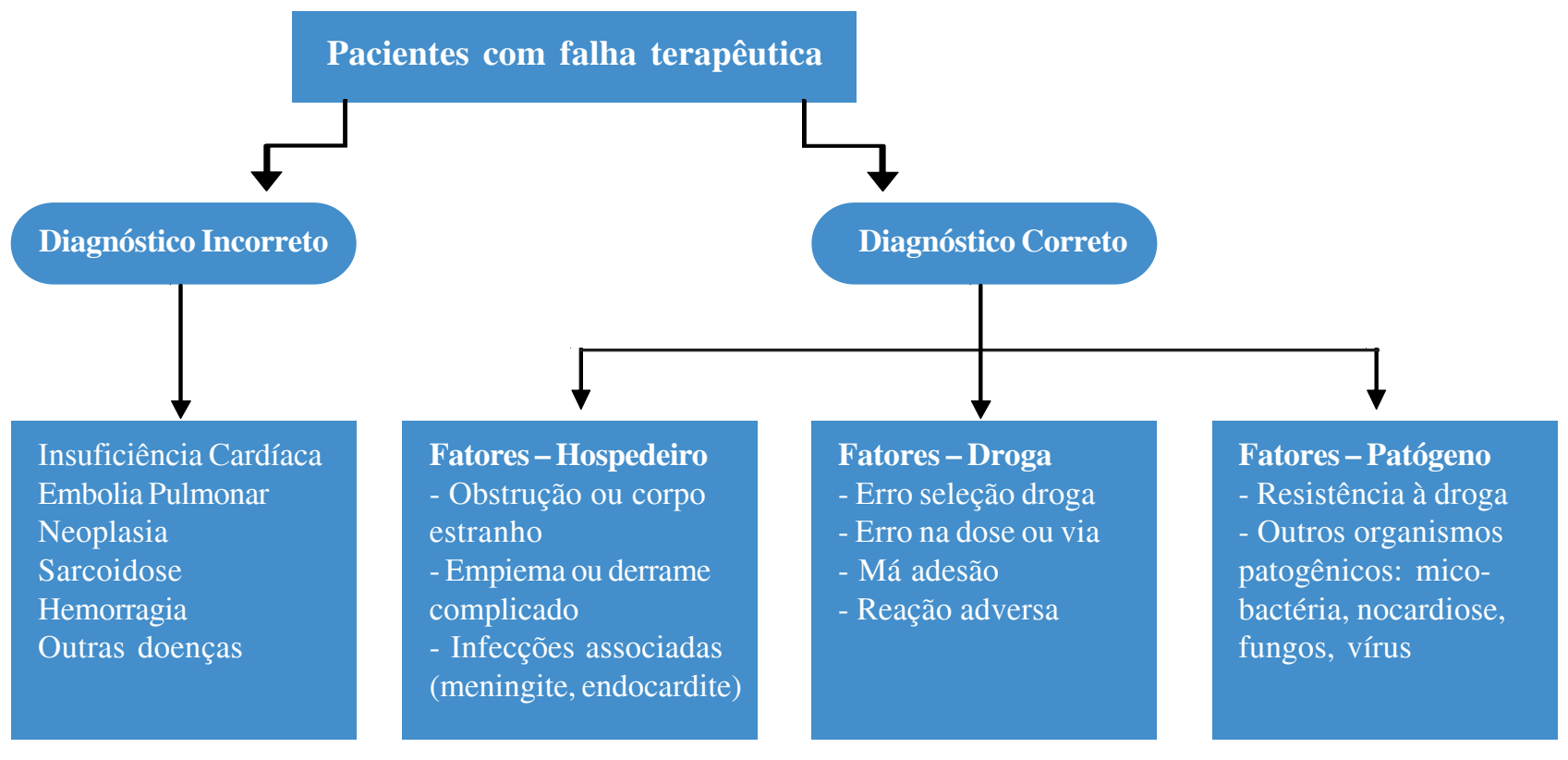

Figura 3. Principais fatores relacionados à falha terapêutica.

monia pneumocócica. Há infecções à distância, como meningite, endocardite, artrite, pericardite, peritonite e derrame pleural complicado ou empiema. Todo paciente com resposta inadequada à terapêutica inicial deve ser submetido a nova radiografia de tórax e reanálise do líquido pleural.

4) Doenças não infecciosas: podem mimetizar quadro pneumônico. Dentre elas: embolia pulmonar, ICC, carcinoma bronquíolo-alveolar, linfoma, hemorragia intrapulmonar, doenças inflamatórias pulmonares, colagenoses, sarcoidose, pneumonites intersticiais e doença pulmonar secundária a medicações.

\section{Prevenção}

De acordo com a Diretriz Americana ${ }^{5}$ as seguintes recomendações para prevenção de pneumonia bacteriana de comunidade devem ser obedecidas:

1) Todas as pessoas acima de 50 anos e/ou em risco para complicações relacionadas a influenza, com contato domiciliar com pessoas de alto risco e profissionais de saúde devem ser vacinados contra influenza anualmente;

2) Vacina antipneumocócica é recomendada em pacientes acima de 65 anos e em pacientes com comorbidades (doença pulmonar, renal, hepática ou cardiovascular crônica, diabetes mellitus, etilismo, asplenia, imunossuprimidos secundários à doença ou medicações)

\section{Pneumonia hospitalar}

\section{Definição}

Pneumonia hospitalar (nosocomial) é definida como a pneumonia que ocorre em até 15 dias após a alta hospitalar ou após 48 horas de uma internação hospitalar - se inferior a 48 horas, os fatores de risco relacionados à pneumonia bacteriana por germe hospitalar são: pacientes hospitalizados por mais de 2 dias nos últimos 90 dias da admissão atual; pacientes residentes em casas de repouso; pacientes que receberam antibioticoterapia endovenosa recentemente e quimioterapia ou curativos nos últimos 30 dias da infecção atual.

\section{Epidemiologia}

Pneumonia nosocomial é usualmente causada por bactérias, sendo a segunda infecção hospitalar mais comum nos EUA e está associada à maior morbimortalidade, maiores custos hospitalares e dias de internação.

O tempo de início da pneumonia é essencial para divisão em duas categorias de pneumonia hospitalar: 1) início precoce (primeiros 4 dias de internação), geralmente com melhor prognóstico e mais provavelmente causada por germes mais sensíveis aos antibióticos; 2) início tardio (mais de 5 dias após a internação), provavelmente relacionada aos patógenos 
multirresistentes, associada à maior morbimortalidade. Fatores de risco para infecção por germe multirresistente podem ser observados na Tabela 6 .

\section{Tabela 6}

Fatores de risco para Pneumonia nosocomial causada por germes multi-resistentes.

- Terapia antimicrobiana nos últimos 90 dias

- Hospitalização atual $\geq 5$ dias

- Resistência aos antibióticos freqüente no hospital

- Presença dos seguintes fatores: hospitalização prévia por mais de 2 dias nos últimos 90 dias da internação atual; residentes em casas de repouso; terapia de infusão domiciliar; diálise crônica; curativos em feridas

- Doença imunossupressora ou terapêutica imunossupressora

\section{Etiologia}

Os patógenos mais comumente envolvidos em pneumonia nosocomial incluem os bacilos gram negativos aeróbios ( $P$. aeruginosa, E. colli, $K$. pneumoniae) e Acinetobacter spp, principalmente quando a pneumonia teve início após 5 dias da internação hospitalar. Quando a pneumonia ocorre nos primeiros 4 dias da internação, os germes mais encontrados são S.pneumoniae, $M$. catarrhalis e $H$. influenzae. Pneumonia devido a S.aureus é mais comum em pacientes diabéticos, vítimas de trauma craniano e internação em UTI. ${ }^{9}$ O perfil de sensibilidade desses germes é variável e depende de cada hospital.

\section{Diagnóstico}

Em pacientes internados, a ocorrência de pneumonia bacteriana hospitalar é frequente e seu diagnóstico correto tem implicação prognóstica. ${ }^{9}$ Mais ainda, é importante utilizar ferramentas diagnósticas capazes de auxiliar na discriminação de fatores de confusão, evitando uso incorreto de antibióticos ou erro diagnóstico (outra infecção ou outra condição clínica como TEP, atelectasia).

Desse modo, de acordo com o Centers for Disease Control and Prevention ${ }^{10}$, os seguintes critérios abaixo são utilizados para o diagnóstico de pneumonia hospitalar:

1. Duas ou mais radiografias de tórax com pelo menos uma das seguintes alterações: a) novo ou progressivo e persistente infiltrado; b) consolidação ou c) cavitação; e
2. Pelo menos uma das seguintes alterações clínicas: a) febre $\left(>38^{\circ}\right)$ sem outra causa relacionada; b) leucopenia ( $<4000 \mathrm{~GB})$ ou leucocitose $(\geq 12.000$ GB); c) alteração do nível de consciência em pacientes $>70$ anos sem outro fator relacionado; e

3. Pelo menos dois dos seguintes critérios: a) início de expectoração purulenta, ou alteração na característica da expectoração, ou aumento na quantidade da secreção respiratória ou na necessidade de aspiração; b) início ou piora de tosse, dispnéia ou taquipnéia; c) ausculta pulmonar compatível; d) piora da troca gasosa, definida como $\mathrm{PaO}_{2} / \mathrm{FiO}_{2} \leq 240$, aumento da necessidade de oxigênio ou necessidade de ventilação não-invasiva.

Todos os pacientes devem ser avaliados quanto à gravidade, condições clínicas associadas e exclusão de outros possíveis sítios de infecção. Se possível, não utilizar radiografia de tórax obtida no leito, dado a pior qualidade e a dificuldade para interpretação - a radiografia de tórax pode auxiliar na identificação de complicações (derrame pleural e cavitações) bem como na gravidade (multilobar). Assim como na pneumonia comunitária, a presença de derrame pleural puncionável demanda toracocentese diagnóstica.

Obter duas amostras de hemocultura, gasometria arterial, hemograma e bioquímica completa que, junto com parâmetros clínicos, auxiliarão na definição de gravidade e na definição do tratamento. Não é objetivo deste capítulo o enfoque nos exames complementares e discussão fisiopatológica.

\section{Tratamento}

O tratamento da pneumonia nosocomial deve ser baseado na gravidade clínica (presença de SIRS, necessidade de ventilação não-invasiva ou invasiva) e na classificação em relação ao tempo de início do quadro (precoce se inferior a 4 dias; tardio se superior a 5 dias) - esses critérios norteiam a antibioticoterapia no sentido de necessidade ou não de cobertura contra germes multirressistentes quando a terapêutica empírica é instituída. Com a evolução e resultado de culturas, o espectro antibiótico deve ser reduzido e focado no germe responsável pelo quadro. O tratamento encontra-se ilustrado nas Tabelas 7 e 8.

Dada a complexidade no tratamento e sua implicação prognóstica, cada vez mais é necessário focar na prevenção da pneumonia nosocomial. Diversas medidas devem ser implementadas, como lavagem de mãos, elevação de cabeceira no leito, retirada precoce de dispositivos (sondas), fisioterapia respiratória, sentar o paciente fora do leito, dentre outras. 


\title{
Tabela 7
}

Tratamento inicial para pneumonia hospitalar em pacientes sem fatores de risco para germes multi-resistentes, de início precoce e sem gravidade

\begin{tabular}{|c|c|}
\hline Patógeno & Antibiótico \\
\hline $\begin{array}{l}\text { S.pneumoniae } \\
\text { H.influenzae } \\
\text { S. aureus meticilina-sensível } \\
\text { Bacilos gram negativos (E.colli, K. } \\
\text { Enterobacter sp, Proteus sp, Serrat }\end{array}$ & $\begin{array}{l}\text { Cefalosporina de } 3^{\circ} \text { geração sem atividade } \\
\text { antipseudomonas ou Fluoroquinolonas ou ampicilina/ } \\
\text { sulbactam }\end{array}$ \\
\hline $\begin{array}{l}\text { Tabela } 8 \\
\text { Tratamento inicial para pneumor } \\
\text { infecção por germe multi-resiste }\end{array}$ & rave ou de início tardio ou se presença de critérios para \\
\hline Patógeno envolvido & Antibiótico recomendado \\
\hline $\begin{array}{l}\text { Patógenos listados da tabela + } \\
\text { Patógenos multi-resistentes: } \\
\text { - Pseudomonas aeruginosa } \\
\text { - Klebsiella pneumoniae (ESBL+) } \\
\text { - Acinetobacter spp } \\
\text { - Staphylococcus aureus (MRSA) }\end{array}$ & $\begin{array}{l}\text { Cefalosporina com atividade antipseudomonas (cefepime, } \\
\text { ceftazidima) OU Carbapenêmico OU Piperacilina- } \\
\text { tazobactam + Fluoroquinolona antipseudomonas } \\
\text { (ciprofloxacino ou levofloxacino) OU Aminoglicosídeo } \\
\text { (amicacina, gentamicina) + Vancomicina }\end{array}$ \\
\hline
\end{tabular}

\begin{abstract}
Community and hospital acquired bacterial pneumonia in adults are increasing public health problems, with numerous hospitalizations annually and frequent causes of morbidity and mortality. Rapid characterization of the clinical picture must be accompanied by a decision regarding the need for patient hospitalization and treatment should be based on severity of presentation and epidemiological aspects. The follow up is essential, both outpatient and in hospital, in keeping with the criteria of treatment failure and need for review of initial treatment. Particularly in hospital-acquired pneumonia (nosocomial), the diagnosis is a challenge and analysis of time of onset is crucial for directing empirical treatment. Emphasis on prevention is an attempt to reduce the frequency of cases.
\end{abstract}

Key words: Pneumonia, Bacterial/diagnosis. Pneumonia, Bacterial/transmission. Cross Infection.

\section{Referências Bibliográficas}

1. Durrington $\mathrm{H}$, Summers $\mathrm{C}$. Recent changes in community-acquired pneumonia in adults. BMJ 2008;336;1429-33.

2. Halm EA, Teirstein AS. Management of community-acquired pneumonia, N Engl J Med 2002; 347: 2039-45.

3. Pereira CAC, et al. Diretrizes para pneumonias adquiridas na comunidade em adultos imunocompetentes. J Bras Pneumol 2004;30(supl 4):S1-S24.
4. File TM. Community-acquired Pneumonia, Lancet 2003 362;1991-2001.

5. Mandell LA, Wunderink RG, Anzueto A, Bartlett JG, Campbell GD, Dean NC et al. Infectious Disease Society of America/ American Thoracic Society Consensus Guidelines on the Management of Community-Acquired Pneumonia in Adults 2007.

6. Van der Eerden MM, Vlaspolder F, de Graaf CS, Groot T, Jansen HM, Boersma WG. Value of intensive diagnostic microbiologi- 
cal investigation in low and high-risk patients with community-acquired pneumonia. Eur J Clin Microbiol Infect Dis 2005;24:241-9.

7. Reed WW, Byrd GS, Gates RH Jr, Howard RS, Weaver MJ. Sputum gram's stain in community-acquired pneumococcal pneumonia. A meta-analysis. West J Med 1996 Oct; 165:197204.

8. Armitage K, Woodhead M. New guidelines for the management of adult community-acquired pneumonia. Curr Opin Infec Dis 2007;20:170-6.

9. Guidelines for the Management of Adults with Hospital-acquired, Ventilator-associated, and Healthcare-associated Pneumonia. Am J Resp Crit Care Med 2005;171:388-416.

10. Tablan OC, Anderson LJ, Besser R, Bridges C, Hajjeh R. Healthcare infection Control Practices Advisory Committee, Centers for Disease Control and Prevention. MMWR Recomm Resp 2004:53:1-36.

11. Flanders SA, Halm EA. Guidelines for community-acquired pneumonia: are they reflected in practice? Treat Resp Med 2004;3:67-77.

12. Niederman MS, Bass JB, Campbell GD, et al. Guidelines for the initial management of adults with community-acquired pneumonia: diagnosis, assessment of severity, and initial antimicrobial therapy. Am Rev Resp Dis 1993;48:1418-26.

13. Bartlett JG, Mundy LM. Community-acquired pneumonia. N Eng J Med 1995;333:1618-24.

14. Ewig S, Ruiz M, Mensa J, Marcos MA, Martinez JA, Arancibia F, Niederman MS, Torres A. Severe community-acquired pneumonia: assessment of severity criteria. Am J Resp Crit Care Med 1998;158:1102-8.
15. Marrie TJ, Lau CY, Wheeler SL, Wrong CJ, Vandervoort MK, Feagan BG. A controlled trial of a critical pathway for treatment of community-acquired pneumonia. JAMA 2000; 283:749-55.

16. Vincent JL, Bihari D, Suter PM, et al.The prevalence of nosocomial infection in intensive care units in Europe: results of the European Prevalence of Infection in Intensive Care (EPIC) Study. JAMA 1995;274:639-44..

17. Andrews CP, Coalson JJ, Smith JD, Johanson WG Jr. Diagnosis of nosocomial bacterial pneumonia in acute, diffuse lung injury. Chest 1981;80:254-8.

18. Rhew DC, Tu GS, Ofman J, Henning JM, Richards MS, Weingarten SR. Early switch and early discharge strategies in patients with community-acquired pneumonia: a metaanalysis. Arch Intern Med 2001;161:722-7.

19. Siegel RE, Halpern NA, Almenoff PL, Lee A, Cashin R, Greene JG. A prospective randomized study of inpatient i.v. antibiotics for community-acquired pneumonia: the optimal duration of therapy. Chest 1996;110:965-71.

20. Auble TE, Yealy DM, Fine MJ. Assessing prognosis and selecting an initial site of care for adults with community-acquired pneumonia. Infect Dis Clin North Am 1998;12:741-59. [Erratum, Infect Dis Clin North Am 2000;14:xi.]

21. Niederman MS, Mandell LA, Anzueto A, et al. Guidelines for the management of adults with community-acquired pneumonia: diagnosis, assessment of severity, antimicrobial therapy, and prevention. Am J Respir Crit Care Med 2001; 163:1730-54. 